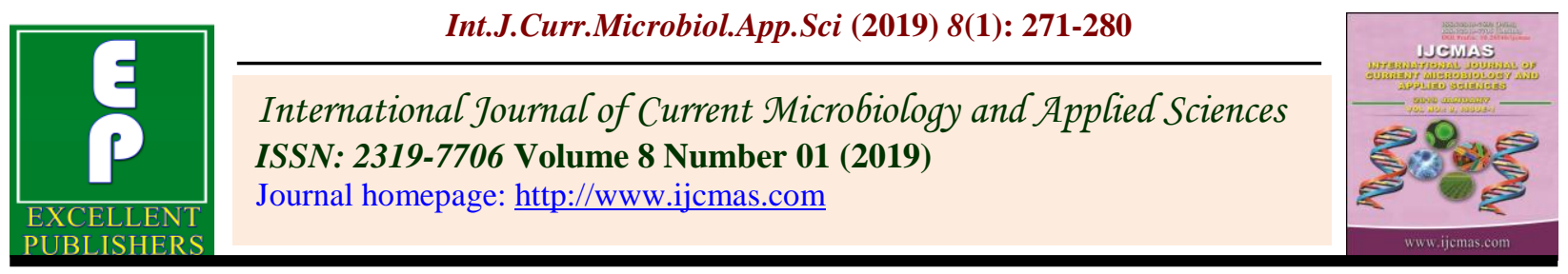

Original Research Article https://doi.org/10.20546/ijcmas.2019.801.030

\title{
Effect of Integrated Nitrogen Management on Rice and Rice Fallow Rabi Crops
}

\author{
M. Latha*, P. Ratna Prasad, P.R.K. Prasad, R. Lakshmipathy and V. Srinivasarao \\ Department of Soil Science \& Agricultural Chemistry, Agricultural College, Bapatla- 522101 \\ Guntur Dt. Andhra Pradesh, India \\ *Corresponding author:
}

A B S T R A C T

\section{Keywords}

INM, Rice, Rice fallow cropping systems, Yield of rice, Rice fallow crops yield and Yield attributes

Article Info

Accepted:

04 December 2018

Available Online:

10 January 2019

\section{Introduction}

Rice based cropping system is a predominant cropping system in coastal Andhra Pradesh. Complementary use of organic and biological sources of plant nutrients along with chemical fertilizer is of great importance for the maintenance of soil health and productivity, especially under intensive cropping system. There is immense need to exploit the alternate source of nutrients viz., organic manure, use of legumes in crop rotation and biofertilizer to sustain the productivity, soil health and soil fertility with more environment friendly nutrient management system.

\section{Materials and Methods}

A field experiment was conducted for two consecutive years (2015-16 \& 2016-17) on clay loam soils of Agricultural College Farm, Bapatla. The experiment was laid out in a two sample t-test for rice in kharif season with 2 treatments and replicated thrice. The treatments consists of $\mathrm{M}_{1} 100 \% \mathrm{RDN}, \mathrm{M}_{2}$ $(50 \%$ RDN $+25 \% \mathrm{~N}$ through FYM $+25 \% \mathrm{~N}$ Through neem cake + Azopsirillum + PSB @ $2.5 \mathrm{~kg} \mathrm{ha}^{-1}$ (INM). During the immediate kharif, the experiment was laid out in a split plot design without disturbing the soil for succeeding rabi crops with the two treatments 
given to kharif rice as main plot treatments and each of these divided into five sub-plots. The experiment was repeated in another field during kharif and rabi seasons. Popular cultivars of rice (BPT 5204), blackgram (PU 31), maize (Sandhya), sorghum (NSH-54), Sunflower (Shreshta) and Mustard (Konark) were used for this study.

The soil is vertisol with bulk density $(1.42 \&$ 1.43 ), porosity (43.50 and $43.80 \%$ ) and water holding capacity $(45.10 \& 45.80 \%)$, slightly alkaline in reaction, $(\mathrm{pH} 7.70$ and 7.50), two fields were non-saline in nature, cation exchange capacity (35.4 \& 37.2), medium range of organic carbon $(0.55$ and $0.50 \%)$. Low nitrogen content (266 and $250 \mathrm{~kg} \mathrm{ha}^{-1}$ ), available phosphorus was (59 and $53 \mathrm{~kg} \mathrm{ha}^{-1}$ ) and available potassium (630 and $668 \mathrm{~kg} \mathrm{ha}^{-1}$ ). The initial soil analysis data is presented in table 1. The present investigation was undertaken during 2015-16 and 2016-17 of consecutive years.

The nutrients were applied through the fertilizers like urea, single super phosphate, muriate of potash. The farm yard manure and neem cake was applied at seven days before transplanting of rice on dry weight basis as per the treatment. The bacterial inoculants applied at the time of sowing as per recommended dosage (Azospirillum + PSB @ $2.5 \mathrm{~kg} \mathrm{ha}^{-1}$ ). The nutrient content in applied organics was given in the table 2 and 3 during first and second year of study, respectively.

The recommended fertilizer doss were applied as 120-40-40, 20-50-0, 200-60-50, 40-40-40, 60-60-30 and 60-60-40 kg N, $\mathrm{P}_{2} \mathrm{O}_{5}$ and $\mathrm{K}_{2} \mathrm{O}$ $\mathrm{ha}^{-1}$ to rice, blackgram, maize, sorghum, sunflower and mustard crops, respectively. The soil samples were analysed as per standard procedures for soil physical and physico-chemical properties. Soil texture by Piper, 1966, bulk density by Dastane, 1967, water holding capacity by Sankaram, 1966, soil reaction and EC by Jackson, 1973, CEC by Bower et al., 1952 and organic carbon by Walkley and Black, 1934. The data obtained during kharif 2015-16 and 2016-17 were analysed statistically using two sample t-test analysis of variance (Panse and Sukhatme, 2000). The t-test value calculated for 12 replications and t-test value was 2.07 . If the $t-$ test value was $>2.07$, it was significant, while $<2.07$ includes non-significant. Whereas the data obtained during rabi 2015-16 and 201617 were analyzed statistically by following split plot design as suggested by Gomez and Gomez (1984). Wherever, the treatment differences were found significant, critical differences were worked out at five per cent probability level and furnished along with mean values of the parameter concerned in tables. Treatmental differences that were nonsignificant were denoted by "NS".

\section{Results and Discussion}

\section{Effect of INM on yield of kharif rice}

Rice yield is presented in table 4 indicated the grain yield of rice during both the years of study was significantly increased due to application of INM over $100 \%$ RDN. Application of organics along with 50\% RDN produced the highest grain yield $(5818 \mathrm{~kg}$ $\mathrm{ha}^{-1}$ ) which was superior over inorganics i.e., $4473 \mathrm{~kg} \mathrm{ha}^{-1}$ during 2015-16 and $5896 \mathrm{~kg} \mathrm{ha}^{-1}$ superior over $100 \%$ RDF i.e., $4598 \mathrm{~kg} \mathrm{ha}^{-1}$ during 2016-17 year. The increment of yield with INM treatment was $30.0 \%$ and $28.22 \%$ during first and second years, respectively. Shanmugan and Veeraputhran (2001), Bhattacharya et al., (2003) also reported beneficial effects of FYM on yield of rice due to better nutrition of crop. The effects of FYM and neem cake were similar and significantly increased the grain yield of rice over control. Application of nutrients like neem cake, different nitrogen levels and biofertilizers had a significant and vital effect on yield and quality attributes of crop. 
Table.1 Initial properties of the experimental soil

\begin{tabular}{|c|c|c|c|}
\hline Particulars & $\begin{array}{c}2015-16 \\
\text { Field } \\
\text { No.13C }\end{array}$ & $\begin{array}{c}2016-17 \\
\text { Field } \\
\text { No.14C }\end{array}$ & Class/ Group \\
\hline \multicolumn{4}{|l|}{ I. Physical properties } \\
\hline \multicolumn{4}{|l|}{ Mechanical composition } \\
\hline 1. Sand $(\%)$ & 42 & 40 & \\
\hline 2. Silt (\%) & 20 & 21 & \\
\hline 3. Clay (\%) & & 39 & \\
\hline Textural class & Clay loam & Clay loam & Clay loam \\
\hline Bulk density $\left(\mathrm{Mg} \mathrm{m}^{-3}\right)$ & 1.44 & 1.43 & Normal \\
\hline Porosity $(\%)$ & 43.50 & 43.80 & Normal \\
\hline Water holding capacity (\%) & 45.10 & 45.80 & Normal \\
\hline \multicolumn{4}{|l|}{ II. Physico-chemical properties } \\
\hline pH (1:2.5) & 7.70 & 7.50 & $\begin{array}{l}\text { Neutral to slightly } \\
\text { alkaline in nature }\end{array}$ \\
\hline$E C\left(d S m^{-1}\right)$ & 0.26 & 0.31 & Non-saline \\
\hline $\begin{array}{l}\text { Cation exchange capacity } \\
\left(\mathrm{cmol}(\mathrm{p}+) \mathbf{k g}^{-1}\right)\end{array}$ & 35.4 & 37.2 & Normal \\
\hline Organic carbon (\%) & 0.55 & 0.50 & medium \\
\hline \multicolumn{4}{|l|}{ III. Available nutrients } \\
\hline $\mathbf{N}\left(\mathrm{kg} \mathrm{ha}^{-1}\right)$ & 266 & 250 & Low \\
\hline $\mathrm{P}_{2} \mathrm{O}_{5}\left(\mathrm{~kg} \mathrm{ha}^{-1}\right)$ & 59 & 53 & High \\
\hline $\mathrm{K}_{2} \mathrm{O}\left(\mathrm{kg} \mathrm{ha}^{-1}\right)$ & 630 & 668 & Very high \\
\hline \multicolumn{4}{|l|}{ IV. Secondary nutrients } \\
\hline $\begin{array}{l}\text { Exchangeable } \quad \mathrm{Ca} \quad(\mathrm{cmol} \\
\left.\mathrm{kg}^{-1}\right)\end{array}$ & 23.39 & 24.07 & Normal \\
\hline $\begin{array}{l}\text { Exchangeable } \mathrm{Mg} \quad(\mathbf{c m o l} \quad(\mathbf{p}+) \\
\left.\mathrm{kg}^{-1}\right)\end{array}$ & 5.80 & 5.70 & Normal \\
\hline $\mathrm{SO}_{4}^{-2}-$ Sulphur $\left(\mathrm{mg} \mathrm{kg}^{-1}\right)$ & 15.00 & 15.50 & Normal \\
\hline \multicolumn{4}{|c|}{ V. Available micro nutrients $\left(\mathrm{mg} \mathrm{kg}^{-1}\right)$} \\
\hline Iron & 27.50 & 25.00 & Sufficient \\
\hline Manganese & 5.50 & 4.90 & Sufficient \\
\hline Zine & 2.55 & 2.65 & Sufficient \\
\hline Copper & 0.59 & 0.65 & Sufficient \\
\hline \multicolumn{4}{|l|}{ VI. Biological properties } \\
\hline $\begin{array}{l}\text { Dehydrogenase activity } \\
\left(\mu \mathrm{g} \text { TPF g } \mathrm{g}^{-1} 2 \mathrm{~h}^{-1}\right)\end{array}$ & 49.14 & 39.15 & Normal \\
\hline \multicolumn{4}{|l|}{ Microbial populations } \\
\hline $\begin{array}{l}\text { Bacteria count } \\
\left(10^{5} \times \text { cfu }^{-1} \text { soil }\right)\end{array}$ & 20.0 & 25.0 & Normal \\
\hline Fungi $\left(10^{3} \times\right.$ cfu g $^{-1}$ soil $)$ & 10.0 & 8.0 & Normal \\
\hline $\begin{array}{l}\text { Actinomycetes } \\
\left(1^{3} \times \text { cfu g }^{-1} \text { soil }\right)\end{array}$ & 15.0 & 15.0 & Normal \\
\hline
\end{tabular}


Table.2 Nutrient content of organics applied during 1styear (2015-16)

\begin{tabular}{|l|c|c|}
\hline Nutrient & FYM & Neem Cake \\
\hline C(\%) & 25.8 & 31.5 \\
\hline Total N (\%) & 0.616 & 4.08 \\
\hline Phosphorus (\%) & 0.24 & 0.21 \\
\hline Potassium (\%) & 0.41 & 0.42 \\
\hline Calcium (\%) & 0.88 & 1.053 \\
\hline Magnesium (\%) & 1.20 & 1.35 \\
\hline Sulphur (\%) & 0.32 & 1.55 \\
\hline Iron (ppm) & 11.72 & 8.11 \\
\hline Manganese (ppm) & 0.534 & 0.862 \\
\hline Copper (ppm) & 0.074 & 0.132 \\
\hline Zinc (ppm) & 0.11 & 0.35 \\
\hline C:N & 41.8 & 7.72 \\
\hline
\end{tabular}

Table.3 Nutrient content of organics applied during 2ndyear (2016-17)

\begin{tabular}{|l|c|c|}
\hline Nutrient & FYM & Neem Cake \\
\hline C(\%) & 24.5 & 29.8 \\
\hline Total N (\%) & 0.58 & 4.89 \\
\hline Phosphorus (\%) & 0.35 & 0.42 \\
\hline Potassium (\%) & 0.48 & 0.43 \\
\hline Calcium (\%) & 0.95 & 1.02 \\
\hline Magnesium (\%) & 1.38 & 1.45 \\
\hline Sulphur (\%) & 0.28 & 1.05 \\
\hline Iron (ppm) & 10.85 & 8.15 \\
\hline Manganese (ppm) & 0.818 & 0.755 \\
\hline Copper (ppm) & 0.080 & 0.115 \\
\hline Zinc (ppm) & 0.15 & 0.78 \\
\hline C:N & 42.24 & 6.09 \\
\hline
\end{tabular}


Table.4 Effect of INM on yield attributes and yield of rice

\begin{tabular}{|c|c|c|c|c|c|c|c|c|c|c|c|c|}
\hline \multirow{2}{*}{ Treatment } & \multicolumn{6}{|c|}{ 2015-16 } & \multicolumn{6}{|c|}{ 2016-17 } \\
\hline & $\begin{array}{l}\text { Productive } \\
\text { tillers } \mathbf{m}^{-2}\end{array}$ & $\begin{array}{c}\text { Filled } \\
\text { grains } \\
\text { per } \\
\text { panicle }\end{array}$ & $\begin{array}{c}1000 \\
\text { seed } \\
\text { weight } \\
(\mathrm{g})\end{array}$ & $\begin{array}{c}\text { Grain } \\
\text { Yield } \\
\left(\mathrm{kg} \mathrm{ha}^{-1}\right)\end{array}$ & $\begin{array}{c}\text { Straw } \\
\text { Yield } \\
\left(\mathbf{k g ~ h a}^{-1}\right)\end{array}$ & $\begin{array}{c}\text { Harvest } \\
\text { Index } \\
(\%)\end{array}$ & $\begin{array}{l}\text { Productive } \\
\text { tillers } \mathbf{~ m}^{-2}\end{array}$ & $\begin{array}{c}\text { Filled } \\
\text { grains } \\
\text { per } \\
\text { panicle }\end{array}$ & $\begin{array}{c}1000 \\
\text { seed } \\
\text { weight } \\
(\mathrm{g})\end{array}$ & $\begin{array}{c}\text { Grain } \\
\text { Yield } \\
\left(\mathrm{kg} \mathrm{ha}^{-1}\right)\end{array}$ & $\begin{array}{c}\text { Straw } \\
\text { Yield } \\
\left(\mathrm{kg} \mathrm{ha}^{-1}\right)\end{array}$ & $\begin{array}{c}\text { Harve } \\
\text { st } \\
\text { Index } \\
(\%)\end{array}$ \\
\hline $\begin{array}{c}M_{1}: \\
\text { RDN }\end{array}$ & 421 & 130 & 14.3 & 4473 & 5607 & 44.37 & 431 & 132 & 14.5 & 4598 & 5657 & 44.83 \\
\hline $\begin{array}{ll}\mathrm{M}_{2}: & \mathbf{5 0 \%} \\
\text { RDN+25\% N - } & \\
\text { FYM+ 25\% N } \\
\text { - neem cake + } \\
\text { bacterial } \\
\text { consortium }\end{array}$ & 433 & 142 & 15.4 & 5818 & 6332 & 47.88 & 449 & 145 & 15.5 & 5896 & 6350 & 48.14 \\
\hline t- value & 2.08 & 6.11 & 3.70 & 7.34 & 3.61 & 3.09 & 2.34 & 6.26 & 3.87 & 8.95 & 3.61 & 2.95 \\
\hline
\end{tabular}

Table.5 Residual effect of INM on yield attributes and yield of blackgram

\begin{tabular}{|c|c|c|c|c|c|c|c|c|}
\hline \multirow[t]{2}{*}{ Treatment } & \multicolumn{4}{|c|}{ 2015-16 } & \multicolumn{4}{|c|}{ 2016-17 } \\
\hline & $\begin{array}{c}100 \text { seed } \\
\text { weight }(g)\end{array}$ & $\begin{array}{c}\text { Seed } \\
\text { yield } \\
\left(\mathrm{kg} \mathrm{ha}^{-1}\right)\end{array}$ & $\begin{array}{l}\text { Haulm } \\
\text { yield } \\
\left(\mathrm{kg} \mathrm{ha}^{-1}\right)\end{array}$ & $\begin{array}{c}\text { Harvest } \\
\text { Index } \\
(\%)\end{array}$ & $\begin{array}{c}100 \text { seed } \\
\text { weight }(g)\end{array}$ & $\begin{array}{c}\text { Seed } \\
\text { yield } \\
\left(\mathrm{kg} \mathrm{ha}^{-1}\right)\end{array}$ & $\begin{array}{l}\text { Haulm } \\
\text { yield } \\
\left(\mathrm{kg} \mathrm{ha}^{-1}\right)\end{array}$ & $\begin{array}{c}\text { Harvest } \\
\text { Index } \\
(\%)\end{array}$ \\
\hline $\mathrm{M}_{1}: 100 \% \mathrm{RDN}$ & 3.98 & 917 & 1437 & 38.95 & 4.55 & 1096 & 1502 & 42.18 \\
\hline $\begin{aligned} \mathrm{M}_{2}: & 50 \% \mathrm{RDN}+25 \% \mathrm{~N}- \\
& \text { FYM+ } \\
& \text { cake + bacterial } \\
& \text { consortium }\end{aligned}$ & 4.17 & 1118 & 1528 & 42.25 & 4.91 & 1210 & 1615 & 42.83 \\
\hline t-value & 9.76 & 14.10 & 5.15 & 2.15 & 8.11 & 4.75 & 12.85 & 2.07 \\
\hline
\end{tabular}


Table.6 Residual effect of INM on yield of maize

\begin{tabular}{|c|c|c|c|c|c|c|c|c|}
\hline \multirow[t]{2}{*}{ Treatment } & \multicolumn{4}{|c|}{ 2015-16 } & \multicolumn{4}{|c|}{ 2016-17 } \\
\hline & $\begin{array}{c}100 \\
\text { kernel } \\
\text { weight(g) }\end{array}$ & $\begin{array}{c}\text { Kernel } \\
\text { yield } \\
\left(\mathrm{kg} \mathrm{ha}^{-1}\right)\end{array}$ & $\begin{array}{c}\text { Stover } \\
\text { yield } \\
\left(\mathrm{kg} \mathrm{ha}^{-1}\right)\end{array}$ & $\begin{array}{c}\text { Harvest } \\
\text { index } \\
(\%)\end{array}$ & $\begin{array}{l}100 \\
\text { kernel } \\
\text { weight } \\
\text { (g) }\end{array}$ & $\begin{array}{c}\text { Kernel } \\
\text { yield } \\
\left(\mathrm{kg} \mathrm{ha}^{-1}\right)\end{array}$ & $\begin{array}{l}\text { Stover } \\
\text { yield } \\
\left(\begin{array}{l}\text { kg ha } \\
1\end{array}\right)\end{array}$ & $\begin{array}{c}\text { Harvest } \\
\text { index } \\
(\%)\end{array}$ \\
\hline $\mathrm{M}_{1}: 100 \% \mathrm{RDN}$ & 26.29 & 6326 & 7411 & 46.00 & 27.96 & 6100 & 7326 & 45.43 \\
\hline $\begin{aligned} \mathrm{M}_{2}: & : 50 \% \mathrm{RDN}+25 \% \mathrm{~N}- \\
& \text { FYM+ } 25 \% \quad \mathrm{~N}- \\
& \text { neem cake + bacterial } \\
& \text { consortium }\end{aligned}$ & 32.30 & 7192 & 8147 & 46.88 & 31.81 & 7682 & 8222 & 48.30 \\
\hline t-value & 6.72 & 5.25 & 7.11 & 2.15 & 2.25 & 16.70 & 13.41 & 3.11 \\
\hline
\end{tabular}

Table.7 Residual effect of INM on yield and yield attributes of sorghum

\begin{tabular}{|c|c|c|c|c|c|c|c|c|}
\hline \multirow[t]{2}{*}{ Treatment } & \multicolumn{4}{|c|}{ 2015-16 } & \multicolumn{4}{|c|}{ 2016-17 } \\
\hline & $\begin{array}{c}1000 \text { grain } \\
\text { weight }(g)\end{array}$ & $\begin{array}{l}\text { Stover } \\
\text { yield } \\
\left(\text { kgha }^{-1}\right)\end{array}$ & $\begin{array}{l}\text { Grain } \\
\text { yield } \\
\text { (kgha })\end{array}$ & $\begin{array}{c}\text { Harvest } \\
\text { Index } \\
(\%)\end{array}$ & $\begin{array}{c}1000 \\
\text { grain } \\
\text { weight }(g)\end{array}$ & $\begin{array}{l}\text { Stover } \\
\text { yield } \\
\left(\mathrm{kg} \mathrm{ha}^{-1}\right)\end{array}$ & $\begin{array}{l}\text { Grain } \\
\text { yield } \\
\left(\mathrm{kg} \mathrm{ha}^{-1}\right)\end{array}$ & $\begin{array}{c}\text { Harvest Index } \\
(\%)\end{array}$ \\
\hline $\mathrm{M}_{1}: 100 \% \mathrm{RDN}$ & 23.90 & 6457 & 2612 & 28.80 & 24.75 & 5906 & 2850 & 31.05 \\
\hline $\begin{aligned} \mathrm{M}_{2}: & 50 \% \mathrm{RDN}+25 \% \mathrm{~N}- \\
& \text { FYM+ } 25 \% \mathrm{~N}-\text { neem } \\
& \text { cake + bacterial } \\
& \text { consortium }\end{aligned}$ & 24.55 & 7127 & 3618 & 33.67 & 29.00 & 8325 & 3750 & 32.50 \\
\hline t- $\quad$ value & 3.05 & 4.86 & 5.44 & 3.05 & 2.55 & 18.36 & 3.73 & 2.35 \\
\hline
\end{tabular}


Table.8 Residual effect of INM on yield of sunflower

\begin{tabular}{|c|c|c|c|c|c|c|c|c|}
\hline \multirow[t]{2}{*}{ Treatment } & \multicolumn{4}{|c|}{ 2015-16 } & \multicolumn{4}{|c|}{ 2016-17 } \\
\hline & $\begin{array}{l}1000 \text { seed } \\
\text { weight }(g)\end{array}$ & $\begin{array}{c}\text { Stover } \\
\text { yield } \\
\left(\mathrm{kg} \mathrm{ha}^{-1}\right)\end{array}$ & $\begin{array}{c}\text { Seed yield } \\
\left(\mathrm{kg} \mathrm{ha}^{-1}\right)\end{array}$ & $\begin{array}{c}\text { Harvest } \\
\text { index } \\
(\%)\end{array}$ & $\begin{array}{c}1000 \\
\text { seed } \\
\text { weight } \\
\text { (g) }\end{array}$ & $\begin{array}{c}\text { Stover } \\
\text { yield } \\
\left(\mathrm{kg} \mathrm{ha}^{-1}\right)\end{array}$ & $\begin{array}{c}\text { Seed yield } \\
\left(\mathrm{kg} \mathrm{ha}^{-1}\right)\end{array}$ & $\begin{array}{c}\text { Harvest index } \\
(\%)\end{array}$ \\
\hline $\mathrm{M}_{1}: 100 \% \mathrm{RDN}$ & 32.5 & 2908 & 1024 & 26.04 & 36.5 & 2712 & 1125 & 29.32 \\
\hline $\begin{aligned} \mathrm{M}_{2}: & \mathbf{5 0} \% \mathrm{RDN}+\mathbf{2 5 \%} \\
& \mathrm{N}-\mathrm{FYM}+\mathbf{2 5 \%} \\
& \mathrm{N}-\text { neem cake + } \\
& \text { bacterial } \\
& \text { consortium }\end{aligned}$ & 42.0 & 3159 & 1507 & 32.30 & 44.8 & 3177 & 1780 & 35.90 \\
\hline t- value & 2.15 & 2.08 & 17.14 & 4.55 & 3.08 & 6.61 & 3.69 & 3.11 \\
\hline
\end{tabular}

Table.9 Residual effect of INM on yield of mustard

\begin{tabular}{|c|c|c|c|c|c|c|c|c|}
\hline \multirow[t]{2}{*}{ Treatment } & \multicolumn{4}{|c|}{ 2015-16 } & \multicolumn{4}{|c|}{ 2016-17 } \\
\hline & $\begin{array}{l}1000 \text { seed } \\
\text { weight }(g)\end{array}$ & $\begin{array}{l}\text { Stover yield } \\
\quad\left(\mathrm{kg} \mathrm{ha}^{-1}\right)\end{array}$ & $\begin{array}{c}\text { Seed yield } \\
\left(\mathrm{kg} \mathrm{ha}^{-1}\right)\end{array}$ & $\begin{array}{c}\text { Harvest } \\
\text { index }(\%)\end{array}$ & $\begin{array}{l}1000 \text { seed } \\
\text { weight }(g)\end{array}$ & $\begin{array}{l}\text { Stover yield } \\
\quad\left(\mathrm{kg} \mathrm{ha}^{-1}\right)\end{array}$ & $\begin{array}{c}\text { Seed yield } \\
\left(\mathrm{kg} \mathrm{ha}^{-1}\right)\end{array}$ & $\begin{array}{c}\text { Harvest } \\
\text { index }(\%)\end{array}$ \\
\hline $\mathrm{M}_{1}: 100 \% \mathrm{RDN}$ & 4.05 & 1515 & 815 & 34.97 & 4.26 & 1619 & 915 & 36.10 \\
\hline $\begin{aligned} \mathrm{M}_{2}: & \mathbf{5 0 \%} \mathrm{RDN}+\mathbf{2 5 \%} \mathrm{N}- \\
& \text { FYM+ } \\
& \text { cake + bacterial } \\
& \text { consortium }\end{aligned}$ & 4.26 & 1805 & 1025 & 36.21 & 4.55 & 1695 & 1135 & 40.10 \\
\hline t - value & 2.11 & 2.09 & 9.45 & 2.55 & 3.08 & 2.13 & 13.34 & 2.15 \\
\hline
\end{tabular}




\section{Residual effect of INM on yields of rabi crops}

Seed yield of blackgram is presented in table 5. Significantly higher seed yield was obtained in residual effect of $\mathrm{M}_{2}$ treatment with values of 1118 and $1210 \mathrm{~kg} \mathrm{ha}^{-1}$ in the year 2015-16 and 2016-17, respectively. These yields were higher by $21.9 \%$ and $10.40 \%$ over $\mathrm{M}_{1}$ during first and second years, respectively, which indicated the residual effect of INM treatment $\left(\mathrm{M}_{2}\right)$ imposed in rice during kharif. Similar results were obtained by Gajendrasingh et al., (2016) who reported that INM had showed the highest seed yield compared to inorganics only.

Residual effect of INM to rice increased the kernel yield of maize by 866 and $1582 \mathrm{~kg} \mathrm{ha}^{-1}$ compared with $100 \%$ RDN $\left(\mathrm{M}_{1}\right)$ in first and second years, respectively. The maize grain yield is represented in table 6 . The kernel yield was obtained $7192 \mathrm{~kg} \mathrm{ha}^{-1}$ during 201516 and $7682 \mathrm{~kg} \mathrm{ha}^{-1}$ in M2 during 2016-17 year. These results were in line with findings of Singh et al.(2000). Experiments performed by Negassa et al., (2001) exhibited that there was significant residual effects of FYM which influenced maize grain yields.

The increase of $38.5 \%$ was obtained during 2015-16 and 31.5\% during 2016-17 in $\mathrm{M}_{2}$ over $\mathrm{M}_{1}$ of sorghum grain yield. The sorghum yield is represented in table 7 . The grain yield of sorghum significantly improved with the application of INM. Organic and inorganic fertilizers are an efficient exogenous source of plant nutrients. When fertilizers are used as balanced fertilizer use, along with complementary use of organic and bio sources can help reverse environmental degradation by providing much needed nutrients to the soil, thereby increasing crop yields (Sudhanshu, 2013). The stover yield of sorghum significantly improved with the application of inorganic fertilizers @ 50\% $\mathrm{RDN}+25 \% \mathrm{~N}$ through FYM $+25 \% \mathrm{~N}$ through neem cake + recommended dose of bacterial inoculants to the preceding rice crop. The increase might be a result of improvement in soil properties in terms of soil, which reflected on increasing biological yield as reported by Kachapur et al., (2001).

Significantly higher grain yields of sunflower were noticed in residual effect of $\mathrm{M}_{2}$ treatment. The increase of $47.16 \%$ was obtained during first year and $58.2 \%$ during second year. Higher biomass production and large canopy spread would have induced the optimally fertilized plants to the increase in seed yield commensurate with large nutrient concentration in seed. Gudade et al., (2011) reported that the seed yield obtained under $100 \%$ RDF + organics was significantly higher than 50\% and 100\% RDF. Application of integrated nitrogen management to rice increased the stover yield of sunflower by $251(8.63 \%)$ and 465 (17.14\%) compared with M1 in first and second years, respectively. The stover yield was increased from $2908 \mathrm{~kg}$ $\mathrm{ha}^{-1}$ to $3159 \mathrm{~kg} \mathrm{ha}^{-1}$ during the year 2015-16 and it was increased from $2712 \mathrm{~kg} \mathrm{ha}^{-1}$ to $3177 \mathrm{~kg} \mathrm{ha}^{-1}$ during 2016-17. The yield of sunflower is represented in table 8 . The increase in stover yield might be due to the fact that the applied FYM released the nutrients were in close conformity with the findings of Nanjudappa et al., (2001) and the applied FYM released the nutrients in adequate amount in the following season also.

Significantly higher seed yields of mustard was noticed in residual effect of $\mathrm{M}_{2}$ treatment. The increase of $25 \%$ was obtained during 2015-16 and 24\% during 2016-17. The mustard seed yield is represented in table 9. Similar results were found by Sonam Lecha, 2015. Thaneswar et al., (2017) exhibited that integrated application of 100\% RDF along with vermicompost @ 5t/ha obtained higher 
seed yield. Combination of organic and inorganic fertilizers was an efficient exogenous source of plant nutrients. When fertilizers were used insufficient and imbalanced, balanced fertilizer use, along with complementary use of organic and bio sources can helped reverse environmental degradation by providing much needed nutrients to the soil, thereby increasing crop yields (Sudhanshu, 2013). Application of integrated nitrogen management to rice increased the stover yield of mustard by 290 $\mathrm{kg} \mathrm{ha}^{-1}$ and $76 \mathrm{~kg} \mathrm{ha}^{-1}$ compared with NPK alone treatment $\left(\mathrm{M}_{1}\right)$ in first and second years, respectively.

In conclusion, plant height, dry matter yield, yield attributes, grain yield and straw yield of rice were recorded significantly higher in the treatment of $\mathrm{M}_{2}(50 \% \mathrm{RDN}+25 \% \mathrm{~N}$ through $\mathrm{FYM}+25 \% \mathrm{~N}$ through neem cake + Azospirillum and PSB @ 2.5 $\mathrm{kg} \mathrm{ha}^{-1}$ ). The increment of yield with INM treatment was $30.0 \%$ and $28.22 \%$ during 2015-16 and 201617 years, respectively. Growing of succeeding rabi crops on yield attributes and yield were significantly influenced by the INM which imposed to preceding rice crop. Plant height, dry matter yield, yield attributes, grain yield, stover yield, test weight and harvest index of rabi crops (blackgram, maize, sorghum, sunflower and mustard) recorded highest in all those plots, which received organics along with 50\% RDN+ Azospirillum and PSB@ $2.5 \mathrm{~kg} \mathrm{ha}^{-1}$ to preceding rice were more than those received $100 \%$ RDN alone. Application of INM to preceding rice crop, increased the all rabi crop yields by $25-30 \%$ when compared to $\mathrm{M}_{1}$.

\section{References}

Bhattacharya, S.P., Sitangshu, S., Karimadkar, A.J., Bera, P.S., Lalitha, M., Sarkar, S and Mandal, L. 2003. Effects of humic acid on the growth and yield of transplanted summer rice. Environment and ecology, 21(3): 680683.

Bower, C.A., Reitemeier, R.F and Fireman, M. 1952. Exchangeable cations analysis of saline and alkali soils. Soil Science, 73: 251-261.

Dastane, N.G., 1967. A Practical Manual for water use research, Navabharat, Prakashan Publication, Poona (India), 120.

Gajendra Singh, Pushkar Choudhary, Bharat Lal Meena, Rajveer Singh Rawat and Bhanwar lal Jat, 2016. Integrated nutrient management in Blackgram under rainfed condition. International Journal of Recent Scientific Research, 7(10): 13875-13894.

Gomez, K. A and Gomez, A.A. 1984. Randomized Block Design in Statistical Procedure for Agricultural Research. Published by a Wiley Inter Science, USA: 621-635.

Gudadhe, N.N., Khnag, V.T., Thete, N.M., Lambade, B.M and Jibhkate, S.B. 2011. Studies on organic and inorganic sources of nutrient application in cotton- chickpea cropping sequence, Omonrice, 18:121-128.

Jackson, M.L. 1973. Soil chemical analysis. Prentice Hall of India Private Ltd., New Delhi. 134-182.

Kachapur, M. D., Roodagi, L. I and Chittapur, B. N. 2001. Influence of vermicompost on the productivity of sorghum. Karnataka Journal of Agricultural Science, 14(3): 779-780.

Nanjundappa, G., Shivaraj, B., Janarjuna, S and Sridhara, S. 2001. Effect of organic and inorganic sources of nutrients applied alone or in combination on growth and yield of sunflower. Helia, 24(34):115-120.

Negassa, W., Negisho, K., Friesen, D. K. Ransom, J and Yadessa, A. 2001. Determination of optimum farm yard 
manure and NP fertilizers for maize on farmers fields. Seventh Eastern and Southern Africa Regional Maize Conference, 387-393.

Panse, V. G and Sukhatme, P. V. 2000. Statistical methods for Agricultural workers. Published by ICAR, New Delhi.

Piper, C. S. 1966. Soil and plant analysis. Hans Publishers, Bombay: 368.

Sankaram, A. 1966. A laboratory manual for agricultural chemistry. Asia publishing House. Bombay. 41-149.

Shanmugam,M and Veeraputhran, R 2001. Effect of organic manure, biofertilizers, inorganic nitrogen and $\mathrm{Zn}$ on growth and yield of rabi rice. Madras Agricultural Journal. 87(1/3):90-93.

Singh, A.K., Amgain, L.P and Sharma, S.K. 2000. Root characteristics, soil physical properties and yield of rice as influenced by INM in rice-wheat system. Indian Journal of Agronomy, 45(2): 217-222.

Sonam Lepcha, Moinuddin, D. R and Kriteshna Bhejel. 2015. Influence of different organic and inorganic sources of nitrogen on growth, yield and oil content of Indian mustard. Journal of international Academic Research for multidisciplinary. 3(11).

Sudhanshu, S. K. 2013. Effect of in-situ soil moisture conservation practices and different nitrogen sources on rain fed sorghum (Sorghum biocolor L. Moensh) and soil properties Ph.D. Thesis Andhra Pradesh, Acharya N. G. Ranga Agricultural University, Hyderabad, Rajendranagar.

Thaneswar Vishram Singh, Jai Prakash, Manoj Kumar, Ateesh Kumar, S and Singh, R. K. 2017. Effect of integrated nutrient management on growth and yield of mustard in irrigated condition of Upper Gangetic plain zone of India. International Journal of Current Microbiology and Applied Sciences. 6(1): 922-932.

Walkley, A. Black, A. 1934. An Examination of degtjareff, method for determining soil organic matter and a proposed modification of the chromic acid titration method. Soil Science. 37: 2938.

\section{How to cite this article:}

Latha, M., P. Ratna Prasad, P.R.K. Prasad, R. Lakshmipathy and Srinivasarao, V. 2019. Effect of Integrated Nitrogen Management on Rice and Rice Fallow Rabi Crops. Int.J.Curr.Microbiol.App.Sci. 8(01): 271-280. doi: https://doi.org/10.20546/ijcmas.2019.801.030 